\title{
High performance 4D tracking with $100 \%$ fill-factor and very fine pitch Silicon detectors
}

\author{
Marco Mandurrino, ${ }^{a}$ Federico Siviero, ${ }^{* a}$ Nicolò Cartiglia, ${ }^{a}$ Marta Tornago, ${ }^{a}$ \\ Marco Ferrero, ${ }^{b}$ Maurizio Boscardin, ${ }^{c}$ Giovanni Paternoster ${ }^{c}$ Francesco Ficorella, ${ }^{c}$ \\ Gian Franco Dalla Betta ${ }^{d}$ and Lucio Pancheri ${ }^{d}$ \\ ${ }^{a}$ INFN, Sezione di Torino \\ Via P. Giuria, 1 - 10125 Torino - Italy \\ ${ }^{b}$ Università degli Studi del Piemonte Orientale \\ Largo Donegani, 2/3 - 28100 Novara - Italy \\ ${ }^{c}$ Fondazione Bruno Kessler \\ Via Sommarive, 18 - 38123 Povo (Trento) - Italy \\ ${ }^{d}$ Università degli Studi di Trento \\ Via Sommarive, 9 - 38123 Povo (Trento) - Italy \\ E-mail: marco.mandurrino@to.infn.it
}

\begin{abstract}
In our contribution we present the performance of RSD (Resistive AC-Coupled Silicon Detectors), an evolution of the LGAD (Low-Gain Avalanche Diode) technology, developed through a collaboration between the Torino division of INFN (the Italian National Institute for Nuclear Physics) and Fondazione Bruno Kessler (FBK), Trento. In this new design, the multiplied charges are slowed down on the detector surface by a resistive $n^{+}$implant and then they induce a signal on the readout metal pads thanks to a dielectric layer, acting as a coupling capacitor. Having a continuous $p$-gain implant over the whole detector area, the RSD technology gets rid of all the isolation structures used to produce standard pixelated trackers by simply transferring the segmentation from the multiplication layer to the readout scheme, given by the AC-pad size and pitch. By properly designing such scheme, we achieved the challenging goal of producing detectors for 4D tracking with very high spatial granularity and $100 \%$ fill-factor while maintaining good timing performances proper of LGAD-based devices (few tens of ps).
\end{abstract}

The 28th International Workshop on Vertex Detectors - Vertex2019

13-18 October, 2019

Lopud, Croatia

* Speaker. 


\section{Introduction}

RSD (Resistive AC-Coupled Silicon Detectors), a new generation of particle trackers with internal gain [1], are specifically intended for high-luminosity environments in near-future HEP experiments, in which Silicon sensors is the enabling technology to perform 4D particle detection [2]. Contrarily to the standard segmented LGAD (Low-Gain Avalanche Diode) [3], where each active area is surrounded by proper isolation implants (see panel (a) of Figure 1) needed to avoid short-circuit and early breakdown at edges, RSD are based on a continuous gain layer. This solution offers the possibility to exploit the maximum geometrical acceptance with a theoretical fill-factor (the ratio between the active and the total area) of $100 \%$. Consequently, the space reconstruction of tracks is obtained through two key elements: (i) a resistive $n$-type implant, slowing down the multiplied charges during their discharge towards the DC contact, and (ii) a coupling dielectric, inducing a capacitive signal on the metal pads located on top of the device (see panel (b) of Figure 1). The RC constant of the readout equivalent circuit determines the waveform shape and - in particular - the discharge time, which is essential to obtain a good timing performance.

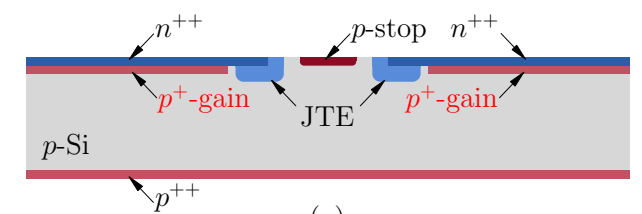

(a)

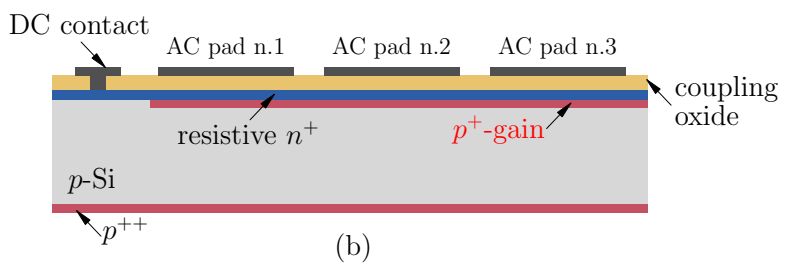

Figure 1: Cross-section of (a) two adjacent active areas in an LGAD-based detector, showing the JTE (Junction Termination Extension) and the $p$-stop segmentation implants, and (b) of an RSD device.

Having an internal gain is beneficial for timing applications because, while the readout amplification would enhance also the electronic noise, the internal charge multiplication mainly provides higher signals. Indeed, when the gain is moderate, the $\mathrm{S} / \mathrm{N}$ (signal to noise) ratio is high and, if the gain increases, the slew rate becomes higher as well. Being the $\mathrm{S} / \mathrm{N}$ and the slew rate directly related to the time resolution $\sigma_{t}$ [4], several simulations and measurements [5] demonstrated that a gain $G \sim 10-20$ in thin sensors $(\sim 50 \mu \mathrm{m})$ allows to reach $\sigma_{t} \sim 30 \mathrm{ps}$ in the detection of minimum ionizing charged particles. Another key aspect characterizing the LGAD technology is its radiation tolerance: as it has been already proven on UFSD (Ultra-Fast Silicon Detectors, i.e. LGAD-based detectors optimized for timing) this technology can operate in radiation-intense environments, with fluence up to $\phi \sim 10^{15} \mathrm{n}_{\mathrm{eq}} / \mathrm{cm}^{2}$ [6], a value which is fully compatible with the expectations for the MIP Endcap Timing Layer (ETL) in the High-Luminosity upgrade of CMS at CERN [7]. RSD are designed to fully exploit the very good timing properties of the LGAD technology [5, 8] without any gain loss due to segmentation structures, which allows to have very fine pitch detectors for high-performance 4D particle tracking. 


\section{RSD1 design and production}

Since high-luminosity conditions require high-resolution particle tagging both in space and in time with the same detector and, at the same time, a geometrical coverage of at least 95\% [7], we simulated, designed, produced and tested a first run RSD1 of particle detectors based on the resistive AC-coupled readout paradigm (see panel (b) of figure 1) with the high spatial resolution and good timing performance characteristic of the LGAD technology.

The optimal set of the physical and technological parameters involved in the RSD signal formation has been determined through both Spice and 3D TCAD (Technology Computer-Aided Design) simulations [9]. Figure 2 shows two examples of TCAD simulations carried out to investigate the coupling between the charges multiplied in the gain layer and the readout by tuning the $n$-cathode depth (left plot) and the dielectric thickness (right plot). In all calculations, a $1 \times 3$ pad RSD structure with $50-\mu \mathrm{m}$-pitch and $V_{\text {bias }}=300 \mathrm{~V}$ has been accounted for, while the signals are obtained by simulating the injection of a MIP (minimum ionizing particle) heavy ion on pad n.1 and acquiring the signals induced on all the three pads (see panel (b) of Figure 1). As one may see, using a deep cathode or a thick dielectric layer (dashed lines) produces lower signals with respect to the standard fabrication rules (solid lines).

(a)

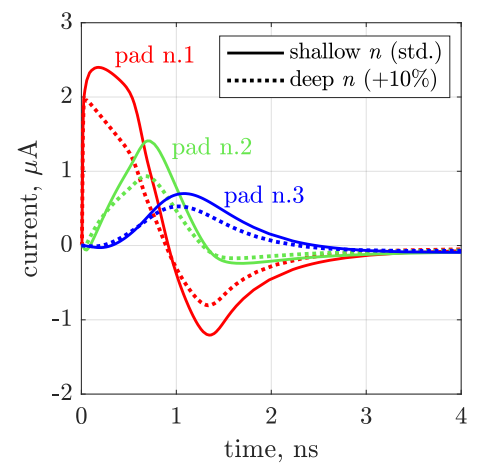

(b)

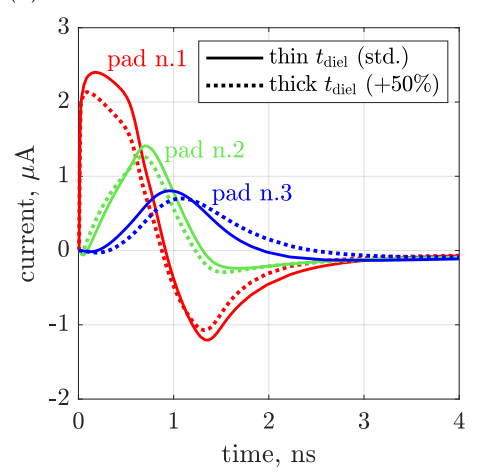

Figure 2: RSD signals simulated with a TCAD tool for a $50-\mu \mathrm{m}$-pitch $1 \times 3$ pad module for a MIP heavy ion passing perpendicularly to pad n.1. The waveforms are calculated by varying the $n$-cathode depth (a) or the dielectric thickness (b) with respect to the foundry's standard values.

With the information obtained through numerical simulations we were able to find the process parameters for the first RSD production run at Fondazione Bruno Kessler (FBK), named RSD1. The batch is fabricated on both Epitaxial (Epi) and Float zone (FZ) 6" wafers with 50- $\mu$ m-thick active Silicon to test the RSD process compatibility with different substrate technologies. As reported in the Table of Figure 3, 15 wafers have been processed by FBK through the step-and-repeat (stepper) lithography, by using different splits of $n^{+}$-resistive dose (increasing letters means increasing implantation dose) as well as the $p^{+}$-gain dose (Boron) and the dielectric thickness. The latter, in particular, has two values, where $\mathrm{H}$ means high and $\mathrm{L}$ stands for low. Furthermore, it is worth noting that the gain dose here has been expressed with the same scale used for standard LGAD-based detectors (for a comparison, see Table 1 in Ref. [6]). 


\begin{tabular}{|c|c|c|c|c|}
\hline Wafer \# & type & $n^{+}$dose & $p^{+}$dose & diel. thickness \\
\hline 1 & FZ & A & 0.92 & $\mathrm{~L}$ \\
2 & FZ & A & 0.94 & L \\
3 & Epi & A & 0.94 & L \\
4 & FZ & A & 0.94 & H \\
5 & FZ & A & 0.96 & L \\
6 & Epi & B & 0.92 & L \\
7 & FZ & B & 0.94 & L \\
8 & FZ & B & 0.94 & L \\
9 & FZ & B & 0.96 & L \\
10 & FZ & B & 0.96 & H \\
11 & FZ & C & 0.92 & L \\
12 & Epi & C & 0.94 & L \\
13 & FZ & C & 0.94 & L \\
14 & Epi & C & 0.96 & H \\
15 & FZ & C & 0.96 & H \\
\hline
\end{tabular}

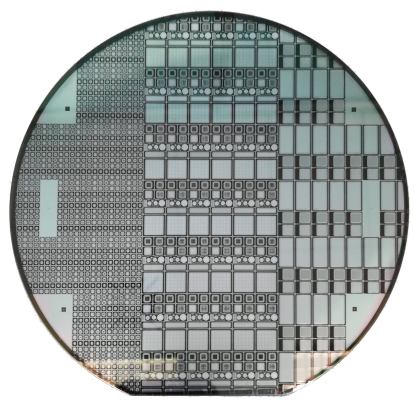

Figure 3: Summary of process split parameters in RSD1 batch (left) and final layout of the same production run at FBK (right).

The RSD1 layout included three lithographic shots, occupying different sectors of the wafer (see the picture in Figure 3). The leftmost block includes detectors made by square matrices of pads with pitch from 50 to $300 \mu \mathrm{m}$ and different pad size. In the central block we implemented $500-\mu \mathrm{m}$-pitch square matrices and some strip modules, while in the last block there are other stripRSD devices for medical applications and two sensors expressly designed on the $64 \times 64$ channels $50 \times 50-\mu \mathrm{m}^{2}$-pitch RD53A chip [10].

\section{Static/dynamic detectors characterization}

The electrical tests performed on several RSD1 samples both in Trento and Torino revealed a very high uniformity for all the implants, both within each wafer and among similar wafers. The breakdown voltage is generally high $(\sim 500 \mathrm{~V})$, it depends only on the gain layer dose and, as a consequence of the above mentioned uniformity, it resulted to be always the same for all the devices of a given wafer. In Figure 4 we reported the $I(V)$ curves measured in several RSD1 samples with different pad pitch/side dimensions coming from wafer 3 (a) and 6 (b) of the RSD1 run. Both plots show that there is a good homogeneity of the $p$-gain implant, since the breakdown voltage is quite constant and does not depend on the detector geometry.

For what concerns the transient properties, unirradiated RSD1 have been tested in the Silicon Laboratory at Università degli Studi di Torino and in the SSD Lab at CERN (EP-DT Department). Both campaigns confirmed that we succeeded in the challenging goal of producing very-fine-pitch Silicon detectors with internal gain and $100 \%$ fill-factor [1]. As an example, the 2D maps of charges obtained with a TCT (Transient Current Technique) [11] setup, and shown in panels (a) and (b) of Figure 5, demonstrate that we have been able to design a correct readout scheme with an effective segmentation down to a nominal pitch of $50 \times 50 \mu \mathrm{m}^{2}$. Indeed, each AC-pad experiences a charge induction which surrounds the pad itself and extends up to the next neighboring pads. This produces a continuous and non-segmented coverage of the detector active area, representing the $100 \%$ fill-factor proper of the RSD readout paradigm. Moreover, since the charge distribution around pads is not constant but changes with position, we are able to reach a space resolution which is better than the nominal sensor pitch. 
(a)

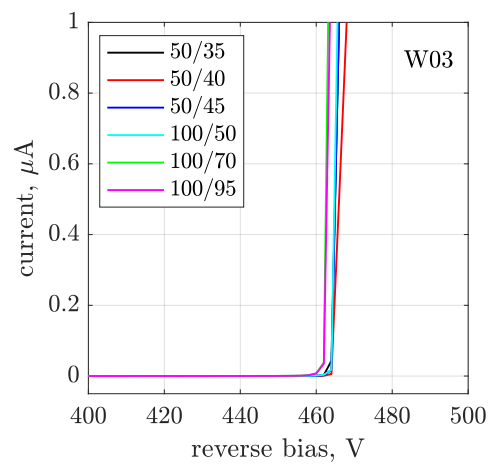

(b)

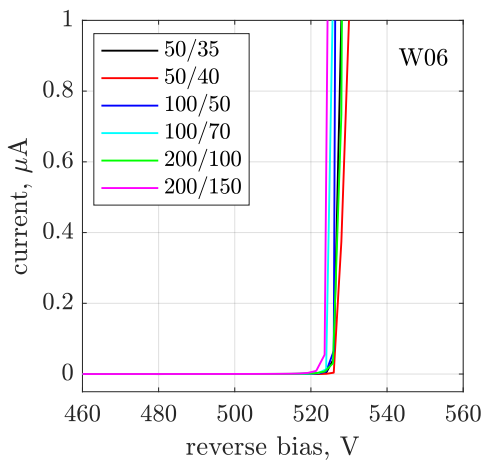

Figure 4: Reverse current-voltage characteristics measured for different sizes of pad (pitch/side) RSD devices coming from wafer 3 (a) and 6 (b). The curves show a good uniformity of the gain implant, reflecting in a very narrow window of breakdown voltages (respectively, 460-464 and 520-526 V).

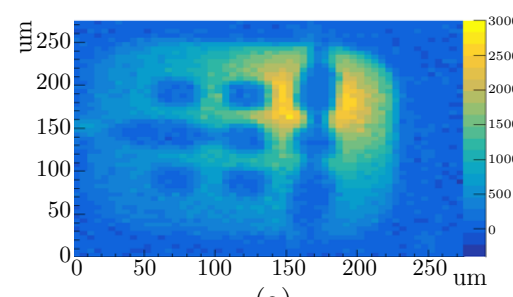

(a)

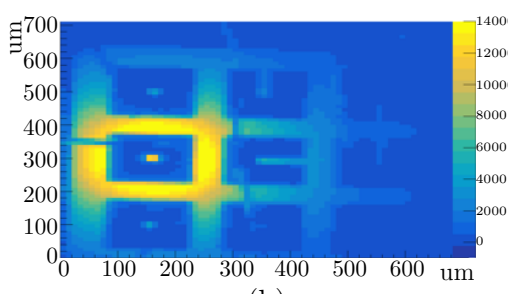

(b)

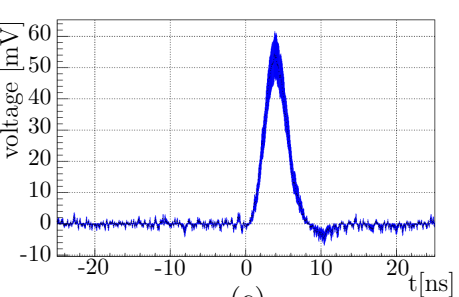

(c)

Figure 5: 2D maps of charges in a 50- (a) and 200- $\mu$ m-pitch (b) square matrix RSD1 sample, respectively from wafer 8 and 10, acquired with the TCT (Transient Current Technique) setup in Torino. Bipolar signal (c) coming from the sensor with pitch $200 \mu \mathrm{m}$. See more details in Ref. [1].

The second important aspect enabling the 4D-tracking is the time resolution of the system. As already stated, the slew rate (and also the rise time) are directly related to the timing performance of a detector. Observing the signal reported in panel (c) of Figure 5, we can see the bipolar nature of waveforms, which is due to the capacitive induction in the first (positive) dominant lobe and determined by the discharge along the resistive layer for what concerns the small (negative) undershoot. Notice that the undershoot here is less prominent than in Figure 2 mainly because of the larger pad area. All the signals acquired with the IR laser of our TCT setup in Torino have shown risetime values ranging from 500 to $700 \mathrm{ps}$, which is perfectly in agreement with the typical behavior of standard LGAD-based trackers. Furthermore, very preliminary measurements with 1MIP IR laser on a $200-\mu \mathrm{m}$-pitch square matrix RSD1 indicated a space and time resolution of, respectively, $\sigma_{s} \sim 5 \mu \mathrm{m}$ and $\sigma_{t} \sim 22 \mathrm{ps}$. But more investigations still have to be carried out in order to find the common performances over all the RSD1 production.

\section{Conclusions}

RSD, an evolution of the traditional LGAD-based Silicon trackers, get rid of any gain loss coming from the segmentation structures used to reach a proper pixel isolation. This is achieved 
through the introduction of a continuous gain layer and by using both a resistive $n$-type implant - slowing down the multiplied charges - and a coupling dielectric, allowing to induce a capacitive signal on the metal pads. In this design, the detector segmentation corresponds to the pads pixellation, while the fill-factor is theoretically $100 \%$.

In this work we presented the design, fabrication and characterization of a first batch RSD1 of detectors by FBK, showing to have achieved the target of producing fine pitch LGAD-based RSD sensors for high performance 4D particle tracking in future high-energy physics experiments.

\section{Acknowledgments}

We kindly acknowledge: INFN Gruppo V, the FBK-INFN collaboration framework, Horizon 2020 Grants no. UFSD669529 and 654168 (AIDA-2020), U.S. Department of Energy Grant no. DE-SC0010107, Dipartimenti di Eccellenza, Università di Torino (ex L. 232/2016, art. 1, cc. 314, 337).

\section{References}

[1] M. Mandurrino et al., "Demonstration of 200, 100, and $50 \mu \mathrm{m}$ pitch Resistive AC-Coupled Silicon Detectors (RSD) with $100 \%$ fill-factor for 4D particle tracking”, preprint on IEEE Electron Device Lett. (2019), DOI: 10.1109/LED.2019.2943242.

[2] M. Mandurrino (on behalf of the RD50 Collaboration at CERN), "Silicon Detectors for the LHC Phase-II Upgrade and Beyond. RD50 Status Report", Connecting the Dots / Workshop on Intelligent Trackers 2019 (CTD/WIT), València (Spain) 2-5 Apr 2019. Available online: arXiv:1910.06045.

[3] G. Pellegrini et al., "Technology developments and first measurements of Low Gain Avalanche Detectors (LGAD) for high energy physics applications", Nucl. Instrum. Methods Phys. Res. A, vol. 765, pp. 12-16, 2014, DOI: 10.1016/j.nima.2014.06.008.

[4] N. Cartiglia et al., "Beam test results of a 16 ps timing system based on ultra-fast silicon detectors", Nucl. Instrum. Methods Phys. Res. A, vol. 850, pp. 83-88, 2017, DOI: 10.1016/j.nima.2017.01.021.

[5] H. F.-W. Sadrozinski, A. Seiden, N. Cartiglia, “4D tracking with ultra-fast silicon detectors”, Rep. Prog. Phys., vol. 81, p. 026101, 2018, DOI: 10.1088/1361-6633/aa94d3.

[6] M. Ferrero et al., "Radiation resistant LGAD design”, Nucl. Instrum. Methods Phys. Res. A, vol. 919, pp. 16-26, 2019, DOI: 10.1016/j.nima.2018.11.121.

[7] CMS Collaboration, "Technical proposal for a MIP timing detector in the CMS experiment Phase 2 upgrade”. Available online: CERN-LHCC-2017-027; LHCC-P-009.

[8] N. Cartiglia et al., "Design optimization of UFSD silicon detectors", Nucl. Instrum. Methods Phys. Res. A, vol. 796, pp. 141-148, 2015, DOI: 10.1016/j.nima.2015.04.025.

[9] M. Mandurrino et al., "Resistive AC-Coupled Silicon Detectors (RSD). A new frontier in high-efficiency 4D particle tracking”, 2018 IEEE Nuclear Science Symposium and Medical Imaging Conference (NSS/MIC), Sydney, Nov 2018.

[10] RD53 Collaboration, “The RD53A Integrated Circuit”. Available online: CERN-RD53-PUB-17-001.

[11] V. Eremin et al., "Development of transient current and charge techniques for the measurement of effective net concentration of ionized charges $\left(\mathrm{N}_{\mathrm{eff}}\right)$ in the space charge region of p-n junction detectors", Nucl. Instrum. Methods Phys. Res. A, vol. 372, no. 3, pp. 388-398, 1993, DOI: 10.1016/0168-9002(95)01295-8. 